\title{
The roles of bottom-up and top-down information in the recognition of reduced speech: Evidence from listeners with normal and impaired hearing
}

\author{
Esther Janse ${ }^{a, b, *}$, Mirjam Ernestus b,c \\ a Utrecht Institute of Linguistics OTS, Utrecht University, Janskerkhof 13, 3512 BL Utrecht, The Netherlands \\ ${ }^{\mathrm{b}}$ Max Planck Institute for Psycholinguistics, PO Box 310, 6500 AH Nijmegen, The Netherlands \\ ' Centre for Language Studies, Radboud University, PO Box 310, 6500 HD Nijmegen, The Netherlands
}

\section{A R T I C L E I N F O}

Article history:

Received 1 July 2009

Received in revised form

23 March 2011

Accepted 25 March 2011

\begin{abstract}
A B S T R A C T
Highly reduced pronunciation variants, such as something like 'yeshay' for yesterday, are abundant in conversational speech. Previous research has shown that listeners understand such pronunciation variants only in their sentential contexts. This study further investigates the roles of the acoustic properties of reduced words themselves and of semantic/syntactic and acoustic information in their contexts. We report five experiments in which participants were tested on the recognition of reduced words originating from a corpus of conversational Dutch. Experiment 1, a visual cloze test, demonstrates that our set of reduced words cannot be guessed just on the basis of their semantic/syntactic context. Experiment 2 replicates the earlier finding that reduced words can only be recognised in their contexts. Experiment 3 shows that the reduced words were less well recognised if the context is presented visually in the form of orthographic transcriptions. These experiments suggest that listeners need some acoustic properties of reduced words themselves, together with semantic/syntactic and acoustic information in their contexts, to recognise reduced words. Experiments 4 and 5 confirm the importance of acoustic information for word recognition by showing that high-frequency hearing loss hinders both the interpretation of the target words' acoustic properties, and the use of neighbouring context.
\end{abstract}

(c) 2011 Elsevier Ltd. All rights reserved.

\section{Introduction}

In conversational speech, words can be reduced quite drastically; for instance, the four-syllable word hilarious can be pronounced as /hleres/ (Johnson, 2004). Importantly, such reductions occur very frequently, especially in highly frequent words or fixed expressions, where three-to-five syllables can be reduced to only one (Ernestus, 2000). Johnson (2004) concluded that over $60 \%$ of the words in his corpus of casual American English speech deviate from their citation forms on at least one segment, and that several segments are absent in $28 \%$ of the word tokens.

Many people are not aware of such reduced pronunciation variants, either in their own speech or in speech of others. They do not notice that segments are missing and tend to report that utterances sound intact. In Kemps, Ernestus, Schreuder, and Baayen (2004) Dutch participants listened to sentences and were asked to monitor for the phoneme $/ 1 /$. Listeners often responded wrongly to reduced forms such as 'eik' / $\varepsilon i k /$ (a reduced form of Dutch / ixəolək/

\footnotetext{
* Corresponding author at: Max Planck Institute for Psycholinguistics, PO Box 310 6500 AH Nijmegen, The Netherlands. Tel.: +3124 3521432; fax: +31243521213.

E-mail address: Esther.Janse@mpi.nl (E. Janse).
}

eigenlijk 'actually'), thereby filling in segments that were not part of the speech signal. Importantly, this happened only if the reduced form was presented in sentence context.

Even though most listeners are not aware of reduced pronunciation variants, there is evidence that reduced speech is more difficult to process than clear speech. Relatively mildly reduced forms (e.g., variants with missing /t/'s or schwas, or variants with flapped /t/'s) produced in isolation are recognised more slowly than their unreduced counterparts (Ernestus \& Baayen, 2007; Janse, 2004; Janse, Nooteboom, \& Quené, 2007; Ranbom \& Connine, 2007; Tucker \& Warner, 2007).

The presence of context has been shown to facilitate the perception of reduced speech substantially. Ernestus, Baayen, and Schreuder (2002) showed that highly reduced word forms, cut from a speech corpus of casual Dutch, were poorly recognised by young normal-hearing listeners when presented as isolated fragments $(52 \%$ correct). They also tested identification of the reduced words if they were presented in their 'Limited context': the target word's adjacent vowels and intervening consonants. Third, identification of the same target forms was also investigated when presented in their full context. The results showed that identification of the highly reduced target words went from $52 \%$ correct in the isolation condition to $70 \%$ in the Limited 
context condition, to $92 \%$ in the Full context condition. Similarly, Pickett and Pollack (1963) showed that very short excerpts from connected speech were often entirely unintelligible, but the same excerpts became intelligible when presented re-embedded in the original context.

The question now arises as to which properties of the context are beneficial. There is no doubt that semantic or syntactic information can aid spoken-word recognition (e.g., McClelland \& Elman, 1986; Norris, 1994; Norris, McQueen, \& Cutler, 2000; Swinney, 1979). Further, several studies have shown that acoustic context cues facilitate recognition of words. Some of these contextual cues are relatively local in the speech signal: if a word's acoustic features spread through coarticulation or assimilation, this will mainly affect its immediately neighbouring segments (Ellis \& Hardcastle, 2002; Gaskell \& Marslen-Wilson, 1998; Gow, 2001, 2002; Nolan, 1992). The same holds for durational cues indicating the location of word boundaries (Salverda, Dahan, \& McQueen, 2003; Shatzman \& McQueen, 2006; Spinelli, McQueen, \& Cutler, 2003), or cues indicating grammatical function (Local, 2003).

There are also acoustic context cues that are located at a greater distance from their source. Cues to the presence of $/ \mathrm{r} /$ in a word can be found a number of syllables before or after the word containing this phoneme (Heid \& Hawkins, 2000; Kelly \& Local, 1986; Local, 2003; Tunley, 1999; West, 2000). Similar longdistance effects were found for the feature [anterior] (Coleman, 2003). Hawkins (2003) argues that this spreading of features over a target word's neighbouring syllables may make the speech signal perceptually coherent.

Crucially, most studies on the role of context, regardless of whether this context mainly contained semantic/syntactic constraints or acoustic cues to the target word, have used carefully lab-recorded speech. The present study investigates the importance of acoustic properties of the word itself, and of information contained in a word's context in the perception of spontaneous rather than careful read speech. Though context plays a crucial role in understanding conversational speech, the very reduction of the context as well as the target that is characteristic of conversational speech may cause specific contextual influences to be less powerful in conversational than in read speech. Conversational speech is often faster than read speech, particularly if we focus on speech fragments containing reduced pronunciation variants. Listeners may not have the time to fully process all information contained in these speech stretches. This may hold for processing of both semantic/syntactic and acoustic information. A second issue is that acoustic cues signalling a target word in conversational speech could be reduced, relative to carefully read speech. Acoustic context cues in casual speech could then be too subtle to be picked up by listeners. The results of Pickett and Pollack (1963) suggest that listeners are able to pick up contextual information in speech that is read aloud fast, but their study did not specify how reduced the fast articulated target words or their contexts were, or which type of information was contained in the context. We specifically focus on stretches of conversational speech containing (strongly) reduced speech, and on the roles of different sources of information in the recognition of reduced words. First, we investigate which information in the words' contexts is used to facilitate target word recognition. Second, we investigate the importance of acoustic properties of the reduced words themselves for spoken-word recognition.

In order to investigate the roles of acoustic (bottom-up) information and semantic/syntactic context (top-down) information in the recognition of reduced words, we followed up the study of Ernestus et al. (2002). We first set out to investigate how semantically/ syntactically constraining the context fragments of their (2002) study were to see which type of context in the (2002) study was particularly beneficial to recognition of the target words. The results of a cloze test on written materials (our Experiment 1) suggest that their 'full context' did not contain many syntactic/semantic cues. In Experiment 2, we replicated the Ernestus et al. (2002) context benefit results with a number of improvements in the experimental design, including only context fragments that are not strongly semantically or syntactically biasing. In Experiment 3, we presented the auditory target words together with a (visual) orthographic transcription of their sentential contexts. This was done to investigate whether the combination of the acoustic properties of the reduced words and the semantic/syntactic information contained in their contexts would facilitate word recognition as much as presenting the targets in their auditory contexts (as was done in Experiment 2). The results showed that providing the visual context also boosted recognition of the (auditorily presented) reduced words, but to a lesser extent than presenting the reduced words in their auditory contexts. Together, these three experiments show that the context effects on target word recognition in Ernestus et al. (2002) should be attributed to the interaction between the acoustic properties of the target word and information contained in the word's context (being both semantic/ syntactic constraints and acoustic cues to the target word).

Experiments 4 and 5 provide initial data about the relevance of the high-frequency part of the spectrum for recognition of target words in and out of their natural contexts. Several studies have shown that while young normal-hearing listeners are hardly aware of the massive reduction in conversational speech style, reduced articulation is much more evident to people with hearing loss, which often affects hearing in the high frequencies in particular (Payton, Uchanski, \& Braida, 1994; Picheny, Durlach, \& Braida, 1985, 1986, 1989; Uchanski, Choi, Braida, Reed, \& Durlach, 1996). This suggests that high-frequency acoustic information may be important for the recognition of conversational speech. Experiment 4 tests this hypothesis directly. In Experiment 4, young normal-hearing listeners were presented with the speech materials as in Experiment 2 , but these materials were filtered with a downward sloping filter, attenuating high frequencies more than low frequencies.

Experiment 5 investigates the recognition of reduced target words in and out of their contexts by older adults. Many older adults suffer from (age-related) hearing impairment that particularly affects the high frequencies. Unlike the young adults tested in Experiment 4, who suddenly had to deal with a speech signal with attenuated high frequencies, older adults may have gradually developed strategies to cope with a more impoverished speech signal. Such strategies may involve a stronger reliance on low-frequency information (e.g., Stelmachowicz, Lewis, Kelly, \& Jesteadt, 1990), or on semantic context (Nittrouer \& Boothroyd, 1990; Pichora-Fuller, Schneider, \& Daneman, 1995). Experiment 5 , therefore, shows how indispensable high-frequency information is for the recognition of our reduced speech materials.

In short, the present study investigates how bottom-up acoustic information in a target word and in its context and top-down semantic/syntactic information in its context contribute to the recognition of that word in conversational speech. These issues are addressed in five experiments: one visual cloze test to test whether our targets' contexts were semantically/syntactically constraining, one study in which auditory presentation of the reduced words was supplemented with a visual (orthographic) presentation of their contexts, and three auditory recognition studies testing how well different groups of listeners recognise the words in and out of their contexts.

\section{Experiment 1: Cloze procedure on sentence contexts}

Experiment 1 investigated whether the context effects on target word identification reported in Ernestus et al. (2002) 
should be attributed purely to strongly biasing semantic or syntactic information in the context. We transcribed the stretches of speech used in Ernestus et al. (2002) orthographically and we presented the context fragments in a visual cloze task. Since participants only received visual information, their guesses on what the target could have been must be based on semantic and/ or syntactic information in the words' contexts and cannot be based on acoustic cues to the target word.

\subsection{Materials and methods}

\subsubsection{Materials}

The test stimuli of the Ernestus et al. (2002) study were drawn from a corpus of Dutch spontaneous casual conversations recorded in a soundproof room (Ernestus, 2000). Each of the 53 stretches incorporated in our study ${ }^{1}$ contains a (reduced) token of 20 different target word types (words or fixed expressions, such as, in ieder geval 'in any case'). The word tokens differed in degree of reduction: degree of reduction was classified as High, Medium, or Low (cf. Section 3.1.1, or Ernestus et al. (2002), for a more elaborate description). An example of the sentence context for the word eigenlijk 'actually' is nou eigenlijk alleen voor deze keer niet 'well actually only for this time not'.

\subsubsection{Participants}

Eighteen young adults (all students at Utrecht University and all native speakers of Dutch) participated in the experiment. The students were paid a small fee for their participation.

\subsubsection{Procedure}

An internet application was made that presented the orthographic transcriptions of all sentence fragments. Target words were replaced by dots. Participants read a sentence fragment and were asked to guess three times which word they thought had been left out. They were encouraged to come up with three different guesses. Only after filling in their three answers for that trial, would they proceed to the next trial. The order in which the 53 sentence fragments were presented was randomised for each participant.

\subsection{Results}

Fig. 1 gives two histograms of the cloze results. Fig. 1a represents how many (out of 18) students guessed the target word correctly as their first guess. Fig. 1b represents how many (again out of 18) participants guessed the word correctly as either the first, second or third guess.

Out of the 53 target words, only 7 items ( 3 items in the High reduction group, 3 items in the Low reduction group, and 1 item in the Medium reduction group) were guessed correctly (either as first, second or third guess) by at least a third (more than 5 out of 18 ) of the participants. This suggests that the context effects in Ernestus et al. (2002) cannot be attributed to strong syntactic and/or semantic constraints.

In the following recognition studies, we analysed only the 46 stimuli that were guessed correctly by less than a third of the participants (and considered the other 7 as fillers). We thus concentrated on the stimuli with minimal semantic and syntactic cues in the context. The remaining items had a mean cloze value of $1.11(S D=1.46)$ (i.e., on average 1.11 out of 18 participants guessed the word correctly).

\footnotetext{
${ }^{1}$ Ernestus et al. (2002) had 54, rather than 53, word tokens, but one token could not be retrieved.
}

\section{Experiment 2: Young normal-hearing listeners}

In the original Ernestus et al. (2002) study, the effect of context on target word recognition may have been confounded with, and possibly inflated by, a number of material and design issues. Therefore, before we follow up on their study and address our experimental questions, we first replicated their study with a number of important changes to see whether we would obtain similar effects. These changes are discussed below. Minor changes are discussed in Section 3.1.

Blocking stimuli by speaker and familiarisation fragment

The stretches of speech in Ernestus et al. (2002) were from 17 different speakers and were presented in random order. Identification of words that are presented in lists has been shown to be worse if the words are from multiple speakers (and are presented in random order) compared to a single speaker, presumably because listeners need time to adapt to each new speaker's characteristics (Sommers, Nygaard, \& Pisoni, 1994). The context benefit in Ernestus et al. (2002) may have been inflated by the continuous switching between speakers, which affects recognition of short fragments more than that of longer fragments. Therefore, in the present study, fragments were blocked by speaker which resulted in 17 speaker blocks with an average length of 3 items (target and filler items). Furthermore, each block of fragments of one speaker was now preceded by a 1-second long familiarisation fragment of this same speaker, drawn from the corpus that the target and filler fragments were drawn from as well. These speaker familiarisation fragments were included to allow speaker adaptation to take place before the test stimuli (targets and fillers) were presented.

\section{Equalised intensity}

In the original (2002) study, intensity differences between speech stimuli were maintained. This could have been partly responsible for the differences in intelligibility between word tokens of different degrees of reduction, since the degree of reduction may naturally covary with signal intensity (the less reduced stimuli being generally louder than the more reduced stimuli). In the present study, we wanted to investigate intelligibility of our target items based on spectral properties of the signal, and on information in their contexts. Mean intensity was therefore equalised over all fragments.

Within-subjects design instead of between-subjects design

In the Ernestus et al. (2002) study, target word tokens were presented in three contexts: in isolation, in limited context (together with adjacent vowels and intervening consonants), and in the sentence fragment they were taken from. Context manipulation in their study was a between-subjects factor: listeners were assigned to one of three context conditions (for all stimuli). In the present study, context was a within-subjects factor to rule out the possibility that differences between conditions are actually differences between participant groups. This is especially important in Experiment 5 where we tested older listeners, since they generally form a much less homogeneous group than young student populations. Furthermore, a within-subjects design also allowed us to relate context use to individual characteristics (such as hearing acuity) of the older participants in Experiment 5.

\subsection{Materials and methods}

\subsubsection{Materials}

Each of the 46 stretches of speech, originating from the Ernestus et al. (2002) study, contains a (reduced) token of one of 20 different 

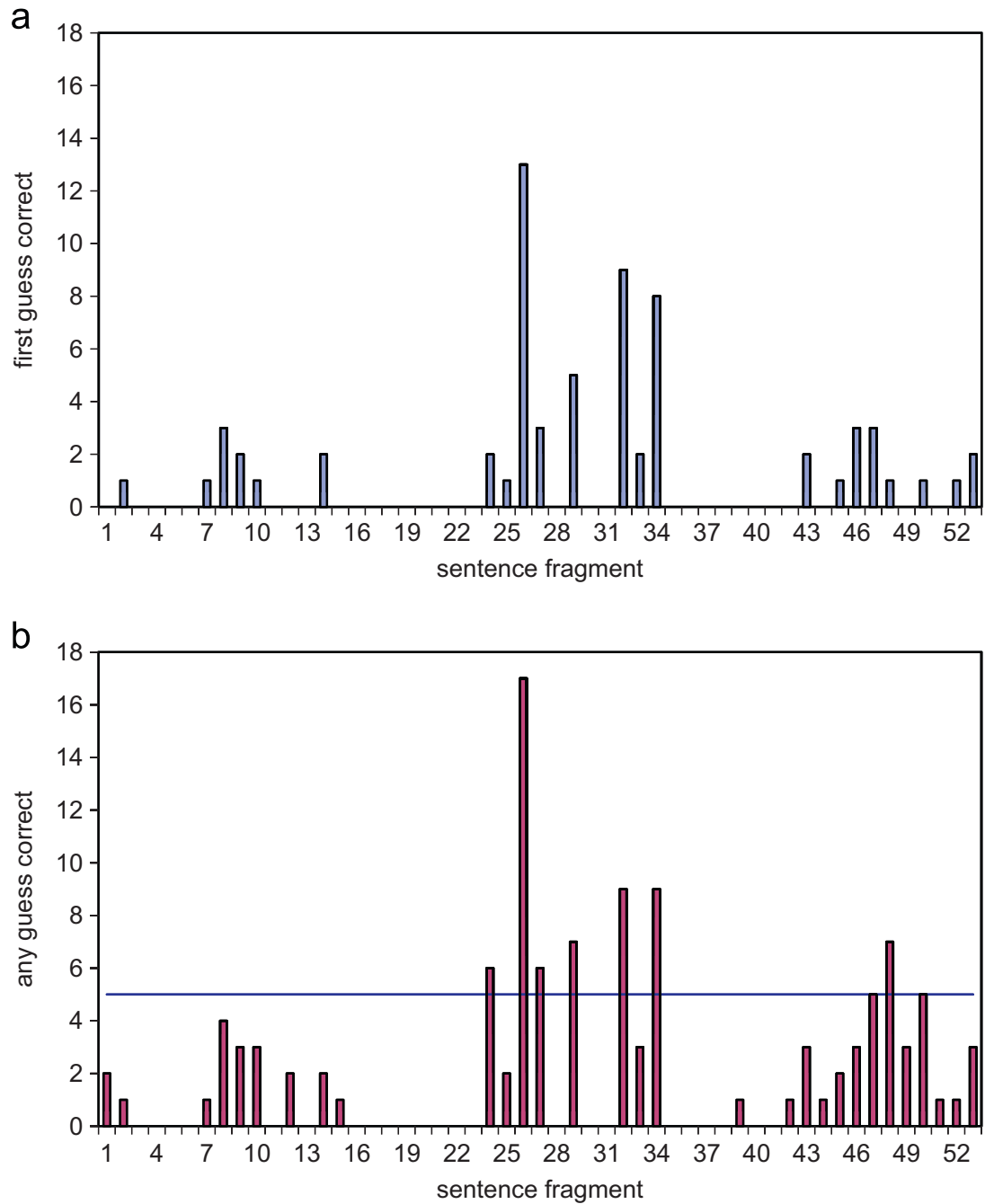

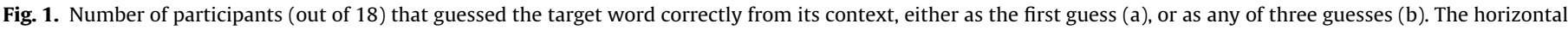
line in the lower graph indicates our cut-off criterion.

target word types (words or fixed expressions, such as, e.g., in ieder geval 'in any case'). As mentioned before, the word tokens differed in degree of reduction. There were tokens with Low (little or no) reduction. Secondly, there were tokens with Medium reduction, missing several segments, but consisting of more segments than the initial, the final, and the stressed segments of their unreduced counterparts, e.g., two-syllabic /'moxək/ instead of the threesyllabic /'moxələk/ mogelijk 'possible.' Third, there were tokens with High reduction, consisting maximally of the initial, final, and stressed segments of their unreduced counterparts, e.g., monosyllabic /'mok/ mogelijk 'possible.' Word tokens were classified on the basis of unanimous phonemic transcriptions of three trained phoneticians who listened to the stretches of speech containing the word forms as well as four preceding and four following syllables. The phoneticians could replay these stretches as often as they thought was necessary for a reliable transcription. For the transcriptions, a segment counted as present if there was an auditory trace (regardless of whether there was clear evidence for this trace in the spectrogram).

In addition to the 46 target items, 62 filler items, which also varied in degree of reduction, were interspersed with the target items. Note that, particularly for the target items, we included multiple tokens of the same word type, e.g., two speakers' realisations of mogelijk 'possible'. Thus, listeners would hear more than one token of the same word type, but always in different degrees of reduction and always from different speakers. The filler items were from the same speakers as the target items and were included to increase the diversity in correct answers from 20 different answers (to the 46 targets) to 75 different answers (to the 46 targets and 62 fillers). Thus, all in all, 108 test fragments (targets and fillers) were presented that elicited 75 different answers.

We tested identification of the test words (targets and fillers) only in sentential context and in isolation. Since there were not sufficient stimuli to present every subject with at least 10 different stimuli in three context conditions per degree of reduction, we restricted the experiment to the two most extreme context conditions in order to determine the maximal effect of context: minimum amount of context (i.e., none) and maximum amount of context (sentence fragment).

\subsubsection{Participants}

Twenty-four young adults, all students at the Radboud University of Nijmegen, participated in this experiment. They were 3 men and 21 women, and were all native speakers of Dutch. They had a mean age of 21 years $(S D=2.1$; range $18-25)$. Their hearing acuity in both ears was tested in a soundproof booth with a Maico ST20 audiometer. Averaged over 1, 2 and $4 \mathrm{kHz}$, mean pure-tone 
threshold in the better ear was $5.6 \mathrm{~dB} \mathrm{HL}(S D=3.7$; range $0-13 \mathrm{~dB}$ ). Mean hearing acuity at several frequencies is graphed in Fig. 6 in Section 6.1.2.

\subsubsection{Design and procedure}

Using a Latin-square design, we rotated context over the 108 test items on two experimental lists. Of each experimental list, three variants were made that varied randomly in the order in which the speaker blocks were presented. This made six different experimental lists to which the participants were randomly assigned.

Participants listened to stretches of speech over headphones (Sennheiser HD 215) in a soundproof booth, with each stretch of speech being presented only once. All speech fragments were presented diotically. Participants were asked to repeat back the speech fragment they heard as accurately and as fast as possible (note again that a sentence fragment either consisted of a word/ fixed expression, e.g., eigenlijk 'actually', or of the word/fixed expression as part of a longer fragment, e.g., nou eigenlijk alleen voor deze keer niet 'well actually only for this time not'). Participants' responses were recorded and scored by the test assistant immediately. The test assistant only scored whether the target word had been reproduced correctly, and ignored whether the context had been repeated correctly. If necessary, the recording could be used later to check a (unclear) response. We chose to elicit spoken responses, since we believe they are easier to provide than written responses, especially for the older participants in Experiment 5. Furthermore, we considered spoken responses to better reflect 'online' processing than written responses because they can be given faster.

All participants were first presented with the sentence condition, then the isolation condition. We know that restoration naturally occurs when listeners are presented with reduced forms in their appropriate contexts (Kemps et al., 2004). In the first part of the experiment the participants were therefore probably unaware of the goal of this study. If the isolated fragments were to be presented first, listeners might know what our research was about and might focus on reduction during the entire study which might result in less natural listening behaviour. Before each context condition, participants were presented with a practice familiarisation fragment and four practice items to get used to the task. In the actual test blocks, each familiarisation fragment was always followed by at least one filler item. Presentation level of the speech stimuli was kept constant for all participants at a mean of $80 \mathrm{~dB}$ SPL (a level which is still comfortable for those with normal hearing).

Finally, in order to have a measure of cognitive performance of the participants, we included a variant of the Stroop colournaming task in our procedure. The Stroop experiment was performed after the listening experiment. Participants were asked to name the colour of a rectangle, which either had the letter string ' $\mathrm{XXXX}$ ' in it in a large white font, or an incongruent colour name (e.g., the word RED in a green or blue rectangle). For each of the three rectangle colours (red, blue and green), there were three pictures (e.g., the red rectangle appeared with the letter strings 'XXXX', 'BLUE' or 'GREEN'). The neutral picture (with the string ' $\mathrm{XXXX}$ ') was presented twice to balance the number of observations per condition (neutral vs. incongruent). These 4 presentations per rectangle colour, times three colours, made 12 trials. These were repeated 10 times in random order, so that there were 120 observations for each participant in total (60 per condition). We measured by means of a voice key how fast people named the colour of the rectangle. The young participants' results on this colour-naming test will be presented together with those of the older adults (in Experiment 5, Section 6.1.2).

\subsection{Results and discussion}

A response was counted as correct if the target word token was reproduced correctly (whether the surrounding words were reproduced correctly was not relevant here). In scoring the responses, we noticed that some listeners would repeat reduced fragments in a reduced manner like the speaker. If the response contained as few or even fewer segments than the presented fragment, we could not be sure that the participant had recognised the fragment correctly or was just repeating what he or she heard without recognising it. We decided to discard these responses, which formed 5\% of the responses, most of which were given in the sentence condition (e.g., the participant's response / $\varepsilon i k /$ was rejected for the target / $/$ ilk/ eigenlijk 'actually', and so was the response /hemə/ for /heməl/ helemaal 'entirely').

In Fig. 2, accuracy rates are given for the word tokens in the two context conditions and the three degrees of reduction (computed as the ratio of the correct responses, relative to the sum of the correct and incorrect responses).

The results (1051 observations) were analysed with a multilevel regression analysis for binomial data (the dependent variable being whether a response was correct or incorrect) with participant and word as crossed grouping factors (Baayen, Davidson, \& Bates, 2008; Pinheiro \& Bates, 2000; Quené \& van den Bergh, 2004). The factors were the Degree of Reduction of the word token (Low, Medium, or High) and whether or not it was presented in Context. In the text below, regression coefficients will be provided for the fixed effects with standard errors in brackets, as well as $z$-values and significance values. The isolation condition with High degree of reduction was mapped on the intercept. Relative to High reduction, items with a Medium degree of reduction elicited higher accuracy scores $(\beta=1.69$ ( $S E=0.27)$, $z=4.79, p<0.001$ ). Similarly, items with Low degree of reduction also elicited higher accuracy scores than the High reduction items ( $\beta=1.69$ ( $S E=0.27), z=6.30, p<0.001$ ). Further, target words were better recognised in sentence context than in isolation ( $\beta=2.69$ ( $S E=0.40), z=6.68, p<0.001)$. The statistical model gave a significantly better fit if Context was added to the random item part, indicating that context was more helpful for some word types than for others (loglikelihood ratio test: $\chi^{2}(2)=9.756$, $p<0.01$ )

The present results look rather similar to those obtained in the Ernestus et al. (2002) study. Most importantly, we verified that presenting target words in their context considerably improves identification performance over presenting them in isolation, even though we only included contexts that are not semantically/syntactically constraining. Furthermore, we now took care

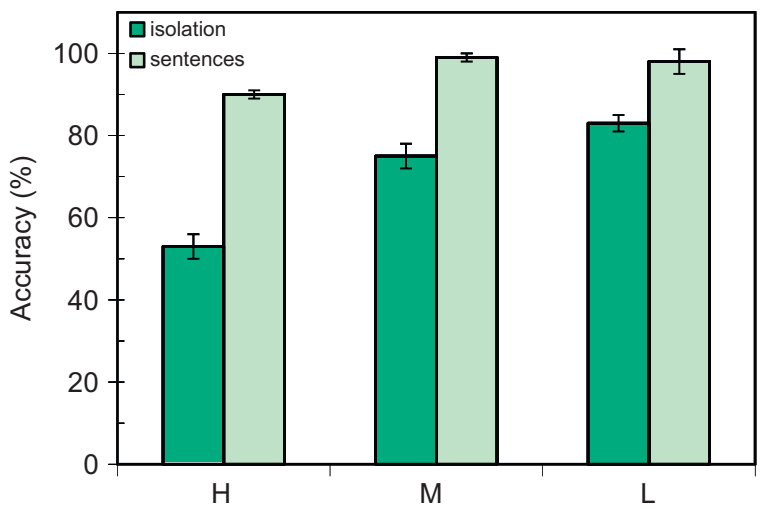

Fig. 2. Results for the young normal-hearing listeners: Accuracy rates for the words of varying degrees of reduction (High, Medium and Low) in the two context conditions (in isolation or in sentences). 
that participants had the chance to adapt to the speaker's characteristics by blocking the target words by speaker and by having every block start with fillers. The current results do not show an interaction between context benefit and degree of reduction, unlike the results in Ernestus et al. (2002). Further analyses showed that this was not due to the exclusion of the items with constraining semantic or syntactic context, but simply to the decreased power as a result of the change from a betweensubjects to a within-subject design (which resulted in half the number of stimuli per condition). We will show that the interaction is statistically significant if we include the other two listener groups (Experiments 4 and 5).

The identification results obtained in Experiment 2 show a robust context benefit effect. Clearly, the context provided by the sentence fragments contains important information to aid identification of the target words: for the word tokens in the High reduction category, identification accuracy went from 53\% in isolation to $90 \%$ in context. This raises the question of what type of information is present in the contexts. Experiment 1 showed that the contexts themselves were not highly semantically/ syntactically constraining. If the auditory context contains acoustic cues to the target word's identity, then the combination of the auditory presentation of the target words with visual orthographic transcripts of their contexts should yield a smaller context benefit than the auditory context benefit observed here in Experiment 2. The effect of visual context on target word recognition was tested in Experiment 3.

\section{Experiment 3: Auditory target words and visual context}

\subsection{Materials and methods}

\subsubsection{Materials}

The auditory target and filler materials were the isolationcondition materials of Experiment 2: participants only heard test items excised out of their contexts. The context manipulation in this experiment entailed whether or not the short auditory test fragments were presented together with a visual orthographic transcript of their sentence contexts.

\subsubsection{Participants}

Twenty-four young adults, all students at the Radboud University of Nijmegen, participated in this experiment. They were 4 men and 20 women, and were all native speakers of Dutch. They had a mean age of 20 years $(S D=2.6$; range $17-26)$. None of them participated in Experiments 1 or 2 . Their hearing acuity in both ears was tested in a soundproof booth with an Oscilla USB-300 screening audiometer. Averaged over 1,2 and $4 \mathrm{kHz}$, mean puretone threshold in their better ear was $0.8 \mathrm{~dB} \mathrm{HL}(S D=5.4$; range -8 to $12 \mathrm{~dB})^{2}$

\subsubsection{Design and procedure}

As in Experiment 2, we rotated context (with or without visual presentation of the sentence context) over the 108 test items on two experimental lists (46 targets and 62 fillers). As in Experiment 2, stimuli were blocked by speaker and each speaker block was preceded by a speaker familiarisation fragment. Further, before each context condition, participants were presented with a practice speaker familiarisation fragment and four practice items to get used to the task. Of each experimental list, three

\footnotetext{
${ }^{2}$ Hearing sensitivity in this group was slightly better than in the young adult group of Experiment $2(t(46)=3.66, p<0.001)$. It is not clear whether this is a true hearing sensitivity difference between groups, or whether this is due to the use of a different screening audiometer.
}

variants were made that varied randomly in the order in which the speaker blocks were presented. This made six different experimental lists to which the participants were randomly assigned.

Participants listened to the short stretches of speech over headphones (Sennheiser HD 215) in a soundproof booth, with each stretch of speech being presented only once. All speech fragments were presented diotically. Participants were asked to repeat back the speech fragment they heard as accurately and as fast as possible. Participants' responses were recorded and scored by the test assistant immediately, following the same procedure as in Experiment 2.

All participants were first presented with the visual-context condition in which they had to combine the auditory presentation of the target with the orthographic presentation of its sentence context displayed on the computer screen. In the last half of the trials, they were presented with the auditory targets in isolation (no context provided). Thus, as in Experiment 2, participants were first presented with the context condition, and then with the isolation condition. The target word's context was presented visually in orthographic form $3000 \mathrm{~ms}$ prior to auditory presentation of the target. As in the visual cloze procedure of Experiment 1, the target word itself was left out of this transcription and was replaced by dots. The orthographic context only disappeared from the screen $2000 \mathrm{~ms}$ after target onset and was therefore visible during presentation of the auditory target. Then, the next trial started.

Presentation level of the speech stimuli was kept constant for all participants at a mean of $80 \mathrm{~dB}$ SPL.

\subsection{Results and discussion}

Scoring of the responses was the same as in Experiment 2. In our scoring procedure, a response to a reduced stimulus was discarded if it contained as few or fewer phonemes than the presented target fragment. This led to the exclusion of $6 \%$ of the responses.

The remaining 1031 observations of Experiment 3 were analysed with a multi-level regression analysis for binomial data (the dependent variable being whether a response was correct or incorrect) with participant and word as crossed grouping factors. The factors were Degree of Reduction of the word token (Low, Medium, or High) and whether or not the token was presented with its visual context. The isolation condition with High degree of reduction was mapped on the intercept. Relative to High reduction items, items with Low degree of reduction elicited higher accuracy scores ( $\beta=0.85$ ( $S E=0.27), z=3.19, p<0.01)$, but there was no significant difference between items of High and Medium reduction. Further, target words were better recognised when their auditory presentation was supplemented with a visual presentation of their context $(\beta=0.72 \quad(S E=0.19), \quad z=3.74$, $p<0.001$ ). There was no interaction between Degree of Reduction and Context.

The results were also compared to those of Experiment 2. Fig. 3 shows the results of the visual-context condition of Experiment 3, together with the results of Experiment 2 (also presented in Fig. 2). This is done for ease of comparison of the auditory and visual context conditions. Accuracy rates are given for the target word tokens in the three context conditions (Experiment 2's isolation and auditory context conditions and Experiment 3's visual-context condition), broken down by the three degrees of reduction of the targets. Accuracy is computed as the ratio of the correct responses, relative to the sum of the correct and incorrect responses.

The results from Experiments 2 and 3 (from both context conditions from both experiments) were compared with a multilevel regression analysis for binomial data. The factors in this 


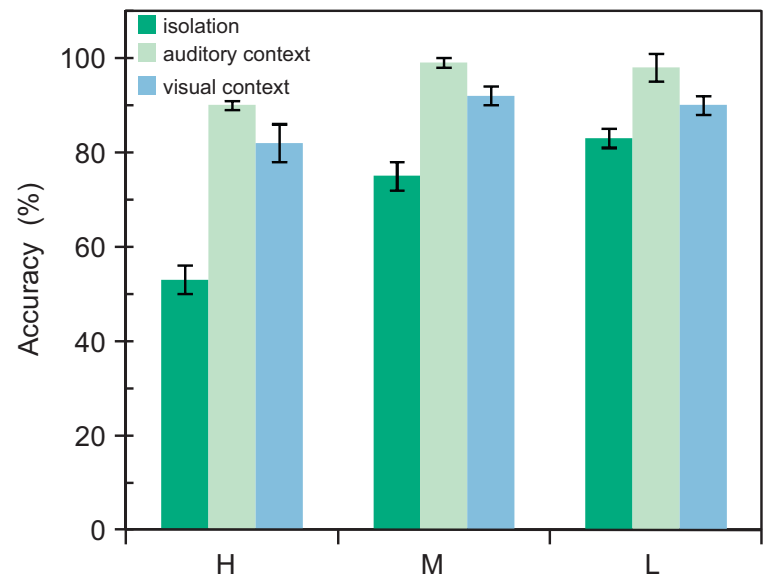

Fig. 3. Results for young normal-hearing listeners: Recognition accuracy rates for the words of varying degrees of reduction (High, Medium and Low) in the three context conditions (in isolation (Experiment 2), with auditory context (Experiment 2), and with visual context (Experiment 3 )).

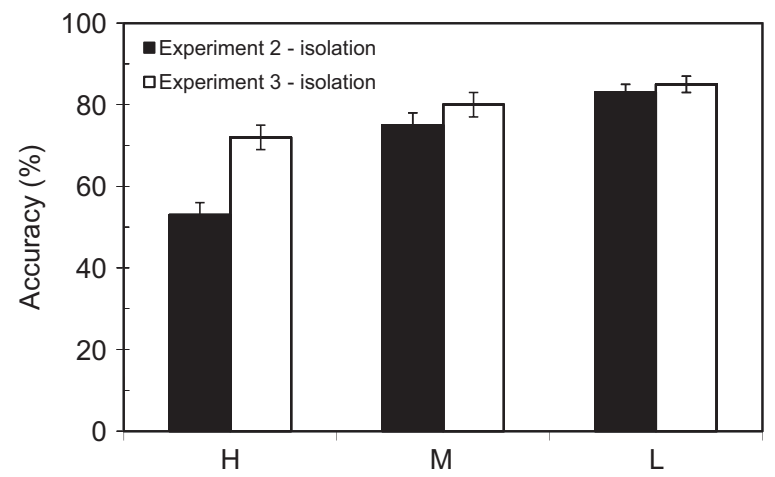

Fig. 4. Recognition accuracy rates for the words of varying degrees of reduction (High, Medium and Low) in the isolation context conditions of Experiments 2 and 3.

analysis were Degree of Reduction of the word token, whether or not it was presented in context (Context Presence), and Experiment (Experiment 2 or 3 ). The best-fitting model showed that there was a significant effect of Context Presence, indicating that words were better recognised when their contexts were available ( $\beta=2.68$ ( $S E=0.27), z=10.09, p<0.001)$. Relative to High reduction items (on the intercept), tokens of Low reduction were better identified $(\beta=1.54 \quad(S E=0.25), \quad z=6.06, p<0.001)$. Likewise, tokens of Medium reduction were better identified than those of High reduction ( $\beta=0.91$ ( $S E=0.26), z=3.45, p<0.001)$. Further, in the experiment with visual context (Experiment 3 ), the difference between items of High and Low reduction was smaller than in the experiment with auditory context $(\beta=-0.66$ $(S E=0.32), z=-2.05, p<0.05)$, as well as the difference in recognition accuracy between items of Medium and High reduction $(\beta=-0.69(S E=0.33), z=-2.09, p<0.05)$. Furthermore, recognition accuracy was overall higher in Experiment 3 than in Experiment $2(\beta=1.00$ ( $S E=0.26), z=3.83, p<0.001)$. This was particularly due to performance differences in the isolation condition across experiments (see Fig. 4). Crucially, the Experiment by Context Presence interaction was significant as well $(\beta=-1.98$ ( $S E=0.32), z=-6.13, p<0.001)$. We investigated this latter interaction in more detail by performing separate subset analyses: first on the trials without context (see Fig. 4), and then on trials with context (see Fig. 3) across the two experiments.

The subset analysis on the isolation-condition trials across experiments (in both experiments, the last half of the trials) showed that items of Low reduction were more accurately identified than items of High reduction $(\beta=1.11 \quad(S E=0.23)$, $z=4.92, p<0.001)$. Perhaps more surprisingly, we also found that recognition accuracy in Experiment 3 was higher than in Experiment $2(\beta=0.62 \quad(S E=0.19), z=3.31, \quad p<0.001)$. One possible explanation for this generally better isolation-condition performance in Experiment 3 may be the slightly better hearing sensitivity of the Experiment 3 participants (cf. Section 4.1.2). This explanation seems unlikely, however, as we checked that individual differences in hearing sensitivity (entered as a covariate) did not predict performance in either Experiment 2 or 3.

Alternatively, the better performance may be due to the fact that participants in Experiment 3 were presented with the reduced tokens excised out of their auditory context throughout the entire study. Even in the condition with visual context (first half of the trials), they only heard the isolated tokens, such that once they were halfway through the experiment (when the isolation condition started), they may have become trained in 'analytical' listening. Furthermore, since the Experiment 3 participants only repeated the targets (without their context) in both context conditions, they may have learned that there was repetition of word forms (remember that the 46 target tokens represented 20 different word types, such that there was repetition of word types across the two context conditions). We investigated learning by adding Trial number to this subset analysis of the isolation-condition trials of Experiments 2 and 3. We expected listeners to improve their recognition performance over trials in both experiments. However, if the Experiment 3 participants have become trained in analytical listening before they started with the isolation-condition trials, they should improve less over trials than the Experiment 2 participants. The results of this analysis (which did not alter the overall result pattern) showed an effect of Trial number, indicating that recognition accuracy indeed improved over trials ( $\beta=0.08$ ( $S E=0.02), z=3.72, p<0.001)$. Trial number also interacted with Degree of Reduction: compared to their improvement on Highly reduced items, participants' performance improved less over trials for the items of Low reduction $(\beta=-0.10$ ( $S E=0.03), z=-3.78, p<0.001)$. Importantly, the Experiment 3 participants tended to improve less over trials than the Experiment 2 participants $(\beta=-0.04(S E=0.02), z=-1.78$, $p=0.07)$, supporting our 'training account' for the better performance of the Experiment 3 participants.

The second subset analysis concerned the results of the auditory and visual context conditions (both presented to participants as the first experiment block). As can be seen in Fig. 3, recognition accuracy was generally lower in Experiment 3's visual context condition than in Experiment 2's auditory context condition $(\beta=-1.39(S E=0.40), z=-3.46, p<0.001)$. This analysis also replicated the effect of Degree of Reduction: both items of Low reduction $(\beta=1.37$ ( $S E=0.34), z=4.00, p<0.001)$, and items of Medium reduction ( $\beta=1.35$ ( $S E=0.39), z=3.43, p<0.001)$ were better identified than items of High reduction.

The higher accuracy rates in the auditory than visual context condition suggest that the auditory context information helps identify the target word. The contextual acoustic cues may involve spreading of features over the target word's neighbouring syllables or may be prosodic cues to the target word's identity. If acoustic information contained in a target word itself and in its neighbouring context is important for target word recognition, the question arises which parts of the spectrum are relevant. We investigated whether decreased sensitivity to high frequencies interferes with identification of the target words both in and out of their auditory contexts. In Experiments 4 and 5 we tested auditory recognition of our target words with two groups of 'hearing-impaired' listeners. 


\section{Experiment 4: Young adults with a simulated hearing loss}

As mentioned in the Introduction (Section 1), hearingimpaired adults with decreased sensitivity for high frequencies are known to have problems understanding conversational speech, which suggests that high frequencies are relevant for the recognition of reduced speech.

Experiment 4 investigated the relative importance of the highfrequency spectrum for the recognition of reduced speech. Participants in this experiment were presented with filtered speech in which the higher frequencies are particularly attenuated.

We did not test actually hearing-impaired young adults because of the risk of potential confounds, such as a somewhat lower education level in the group with hearing problems (relative to our normal-hearing students of Study 2), or a more limited vocabulary knowledge or receptive command of spoken Dutch.

\subsection{Materials and methods}

\subsubsection{Materials}

The materials were the same as those used in Experiment 2 (cf. Section 3.1.1), but the speech stimuli were filtered with a lowpass filter. This filter left frequencies below $1 \mathrm{kHz}$ unaffected and had a $-20 \mathrm{~dB} /$ octave dampening above $1 \mathrm{kHz}$ (see filter function in Fig. 6 in Section 6). ${ }^{3}$ This filtering was a simplified approximation or simulation of age-related hearing loss, and does not mimic the distortion caused by sensorineural hearing loss (cf. e.g., Hargus and Gordon-Salant (1995) and Humes, Dirks, Bell, and Kincaid (1987) for noise-masking as a more accurate method to simulate hearing impairment in normal-hearing listeners). Since we were mainly interested in the effect of attenuation of the high frequencies, we opted for this simple filtering approach (we will come back to this in Section 6).

\subsubsection{Participants}

Twenty-four students from the Radboud University Nijmegen, 23 women and 1 man, were paid to participate in the experiment. They were all native speakers of Dutch and their mean age was 21 years $(S D=2.0$, range $18-27)$. None of them participated in Experiments 1, 2 or 3. Their mean pure-tone average (PTA) over 1,2 , and $4 \mathrm{kHz}$ in the better ear was $6.0 \mathrm{~dB}(S D=4.5)$. There was one student with a PTA of $25 \mathrm{~dB}$, and one with a PTA of $12 \mathrm{~dB}$, but all other students had mean PTAs below $7 \mathrm{~dB}$ (we verified that inclusion of these two participants did not significantly lower the group's recognition performance). Hearing sensitivity in this group did not differ significantly from that in the young adult group of Experiment $2(t(46)<1$, n.s.). Mean hearing thresholds in the better ear of participants in this group are given in Fig. 6 . The filter itself is also graphed in Fig. 6.

\subsubsection{Procedure}

The procedure was the same as in Experiment 2. Filtering had brought the mean intensity of the clear stimuli, equalised at $70.0 \mathrm{~dB}$, to $69.8 \mathrm{~dB}$. As in the previous experiments, stimuli were presented at $80 \mathrm{~dB}$ SPL.

\subsection{Results and discussion}

As in Experiment 2, we only analysed responses that we knew were either correct or incorrect. In Fig. 5, accuracy rates of these

${ }^{3}$ PRAAT software was used (www.praat.org) and Paul Boersma is gratefully acknowledged for his help with the PRAAT filter script: Filter (formula)... self* if $x<1000$ then 1 else $(x / 1000)^{-\log 2(10)} f i$

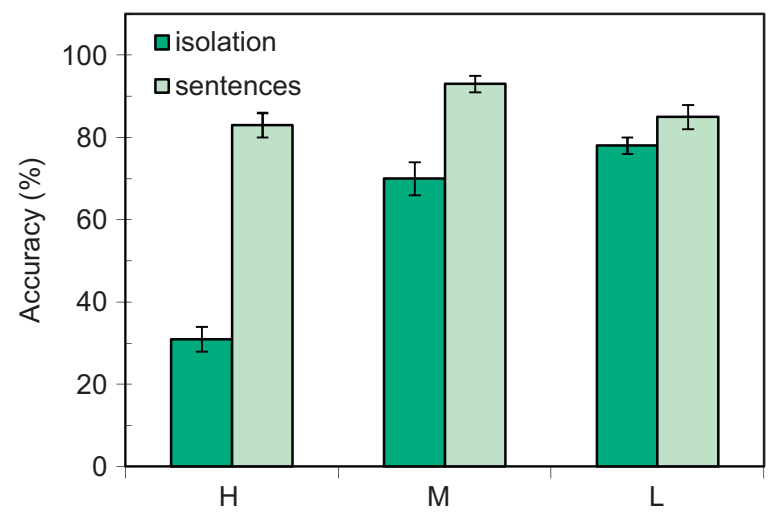

Fig. 5. Results of young adults with simulated hearing impairment: Accuracy rates for the word tokens of varying degrees of reduction (High, Medium and Low) in the two context conditions.

young listeners with simulated hearing impairment are given for the word tokens in the two context conditions (as ratios of the correct responses, relative to the sum of the correct and incorrect responses), broken down by degree of reduction.

The results of the young listeners with simulated hearing impairment were compared to the results obtained in Experiment 2 with normal-hearing young adults. Accuracy results of the two young adult groups were analysed as in the previous study (with Filtering of the stimuli as a between-subjects factor). Again, word tokens were identified better in sentence context than in isolation ( $\beta=2.79$ ( $S E=0.41), z=6.88, p<0.001)$. Overall, the less reduced the target word token, the higher the identification rates: relative to the items of High reduction, items with Medium degree of reduction had higher accuracy $(\beta=1.91 \quad(S E=0.28), \quad z=6.79$, $p<0.001)$, and so had items with Low degree of reduction ( $\beta=2.56$ ( $S E=0.29), z=8.92, p<0.001)$. The effect of Filtering was significant, which shows that overall performance was better in the group who got the clear speech signal $(\beta=1.19(S E=0.27)$, $z=4.41, p<0.001$ ), relative to the filtered group (on the intercept). The interaction between Context and Degree of Reduction was now significant, indicating that context was more beneficial the more reduced the word, replicating the findings by Ernestus et al. (2002). Highly reduced items and items with medium reduction benefited equally from context, but items with a Low degree of reduction benefited less from context $(\beta=-2.19$ $(S E=0.43), z=5.07, p<0.001)$. Most importantly, there was a three-way interaction between Context, Degree of Reduction and Filtering. The young listeners who got the clear signal benefited more from context for the words with Medium reduction $(\beta=2.72$ $(S E=1.19), z=2.28, p<0.05)$, and for the words with Low reduction $(\beta=2.49(S E=0.82), z=3.04, p<0.01)$, than the young participants who listened to the filtered signal.

This three-way interaction was confirmed in item subset analyses (testing whether there was a Filtering by Context interaction for the three degrees of reduction separately). For the items of Low and Medium reduction, Filtering and Context showed a significant interaction: for both subsets, the young adults presented with filtered speech benefited less from context than the young adults presented with the unfiltered signal. For the items in the High reduction group, two groups of young adults benefited equally from context. The statistical model gave a significantly better fit if Context was added to the random item part, indicating that context was not equally helpful for all word types (loglikelihood ratio test $\chi^{2}(2)=34.374, p<0.001$ ).

These results are in line with the hypothesis that highfrequency acoustic information plays an important role in the recognition of reduced speech. Clearly, bottom-up interpretation 
of the acoustic signal is affected by attenuation of the high frequencies: recognition accuracy in the most difficult condition (highly reduced words presented in isolation) dropped from 53\% correct in Experiment 2 to 31\% correct for the young adults who were presented with the filtered speech. Furthermore, these listeners are generally less able to benefit from information contained in a word's context. Attenuation of the high frequencies may degrade specific cues to the target word, and/or may simply weaken the contextual semantic/syntactic constraints through lowered intelligibility of the neighbouring words. It is surprising, however, that the context benefit effect is equal in size for the participants listening to the filtered speech and those listening to the unfiltered speech for the highly reduced words. We will come back to this in the Section "General Discussion".

\section{Experiment 5: Older listeners with hearing impairment}

In Experiment 4, participants were not used to listening to this type of 'muffled'-sounding speech. As a consequence, they may not have been able to compensate for the attenuation of the high frequencies with acoustic information present in the low frequencies or with semantic information. In Experiment 5, recognition of reduced words by older adults with varying degrees of hearing impairment was tested. Age-related hearing impairment is gradual and progressive, such that older adults may develop strategies to cope with a more impoverished speech signal. Such strategies may involve a stronger reliance on acoustic information that is not so much affected by age-related hearing loss (such as low-frequency information) and on semantic context (Nittrouer \& Boothroyd, 1990; Pichora-Fuller et al., 1995). This experiment thus shows whether high-frequency information is as indispensable for target recognition as suggested by Experiment 4 .

Older adults, however, may differ from young adults in more aspects than their hearing sensitivity. Age-related hearing problems involve more than loss of sensitivity, such as decreased temporal and frequency selectivity. The filtering we applied to our materials in Experiment 4 does not mimic the distortion caused by a sensorineural hearing loss. Such signal-distortion aspects of auditory aging may be responsible for the finding that, even when older adults still have relatively good hearing, they require a greater amount of word onset for correct identification of carefully pronounced words than young adults in a gating procedure (Craig, 1992; Craig, Kim, Rhyner, \& Chirillo, 1993). Further, speech comprehension may be affected by age-related cognitive decline (Janse, 2009; Jerger, Jerger, \& Pirozzolo, 1991; van Rooij \& Plomp, 1990, 1992; van Rooij, Plomp, \& Orlebeke, 1989). Among older adults, performance on speech perception tasks has been related to processing speed (Janse, 2009; van Rooij et al., 1989), measures of verbal working memory (e.g., Humes, Lee, \& Coughlin, 2006) and executive control (Tun, O'Kane, \& Wingfield, 2002). Given these differences between young and older adults in auditory processing and in cognitive performance, equal recognition performance for the older adults and the young adults tested in Experiment 4 may still indicate that the older adults use compensation strategies to arrive at this performance level.

Since age group differences in information processing speed (Salthouse, 1996) or attention capabilities may be partially responsible for potential performance differences between the older adults of Experiment 5 and the young adults of Experiments 2 and 4, we investigated how the older group compared to the young adults in terms of cognitive performance. Further, among the older adults, we investigated how individual recognition performance and context use relate to individual listener characteristics, such as hearing acuity, age and measures of cognitive performance.

\subsection{Materials and methods}

\subsubsection{Materials}

The materials were the same as those used in Experiment 2 (cf. Section 3.1.1).

\subsubsection{Participants}

Thirty older adults, all native speakers of Dutch, were recruited via Hoger Onderwijs voor Ouderen ('Higher education for older adults') at the Radboud University of Nijmegen. This organisation provides academic courses on various topics at universities in the Netherlands for people over 55 years of age. People who had subscribed for a course received an information letter in which they were asked to participate in the study. Given the academic level of the courses, the older group was a good match to the young (student) groups of Experiments 1-4 in terms of education level. The older participants were 13 men and 17 women, and had a mean age of 72 years $(S D=5.3$; range $61-84)$.

Mean pure-tone thresholds (with standard error bars) for the older participants' better ear are given in Fig. 6. Averaged over 1, 2 and $4 \mathrm{kHz}$ in the better ear, mean pure-tone average of the older adults was $26.8 \mathrm{~dB} \mathrm{HL}(S D=15$; range $5-66 \mathrm{~dB})$. Average hearing sensitivity in the older adults sample was poorer than the hearing loss simulated in the young adults in Experiment 4: the filtering caused a simulated hearing loss (averaged over 1, 2, and $4 \mathrm{kHz}$ ) of $20 \mathrm{~dB} \mathrm{HL}$. We will come back to this difference in hearing sensitivity in the discussion of the combined results (Section 6.2). Hearing loss, if present, in this participant group was most likely mainly due to age effects: participants had not encountered hearing problems before the age of 50 . Nevertheless, on the basis of the audiogram alone, we cannot rule out that some adults may have encountered some noise-induced hearing loss earlier in life or other pathologies affecting their hearing. Out of the 30 participants, two had a hearing aid in one ear, and two had hearing aids in both ears. These four participants were asked to participate in the listening study without their hearing aids to see how they performed in an 'unaided' situation. By-subject analysis of performance showed that these four older adults did not behave differently from the other older participants.

As mentioned, we included a variant of the Stroop colournaming task in our procedure to have a measure of cognitive performance. Our choice for this task was based on the study by Sommers and Danielson (1999). These authors investigated the identification of words that varied in neighbourhood density in

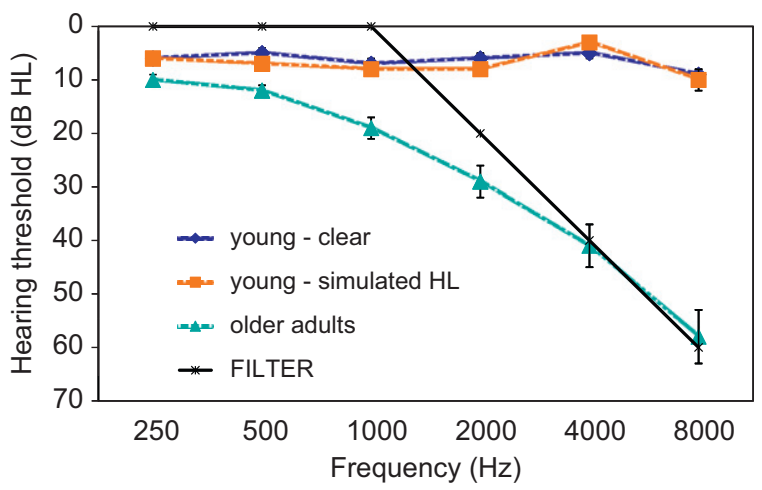

Fig. 6. Average hearing loss in the better ear (pure-tone thresholds in $\mathrm{dB} \mathrm{HL}$ ) for the three listener groups in Experiments 2, 4 and 5 (error bars represent one standard error). The low-pass filter function for the hearing loss simulation of Experiment 4 is also graphed. 
young and older adults. Importantly, they found that older adults' susceptibility to interference in two Stroop-like tasks predicted their ability to identify 'hard' words, that is, words with many similar-sounding neighbours. The correlation was particularly strong for 'hard words' embedded in low-predictability, rather than high-predictability, sentences. Our speech materials have relatively weak semantic constraints and poor intelligibility, and therefore also present a situation with strong lexical competition. Unlike Sommers and Danielson (1999), who opted for auditory variants of interference tasks (their 'inhibition index' was based on a modified version of the selective attention paradigm by Garner (1974) and on an auditory Stroop paradigm), we investigated whether a measure of interference obtained in the visual domain would be predictive of speech perception in our study. If an interference measure obtained in a non-auditory modality is predictive of speech perception performance, it is more likely to be related to general cognitive, rather than auditory, performance.

In our variant of the classic Stroop task (Stroop, 1935), we measured by means of a voice key how fast participants named the colour of the rectangle in a neutral condition (the rectangle contained the letter string ' $\mathrm{XXXX}$ ') and in an incongruent condition in which the coloured rectangle contained an incongruent colour name (e.g., a blue rectangle containing the word 'RED' or 'GREEN'). There were 60 observations for every participant in each of the two conditions. In the neutral condition, mean colournaming time for the older adults was $608 \mathrm{~ms}(S E=12)$ and 599 for the young adults $(S E=12)$ in Experiments 2 and 4 . In the incongruent condition, mean naming time (of the correct colour) was $719 \mathrm{~ms}(S E=13)$ for the older adults and $672 \mathrm{~ms}$ for the young adults $(S E=12)$. Analysis of naming times showed a significant effect of Congruency $\left(F_{1}(1,76)=356.3, p<0.001\right)$, but no effect of Age Group, and an interaction between Congruency and Age Group $\left(F_{1}(1,76)=11.43, p<0.001\right)$. This suggests that the older adults experienced greater interference from the incongruent colour names than the young adults.

We investigated whether differences among the older adults on this interference measure are associated with their individual ability to understand reduced words out of context or to make efficient use of contextual cues. A participant's colour-naming speed in the neutral condition was considered a measure of information processing speed. Naming speed in the incongruent condition, a measure of interference, was considered as a predictor as well, and so was a combined measure of the two conditions of a participant's performance (naming speed in incongruent condition divided by this participant's naming speed in neutral condition, as a normalised measure of interference). Because these three measures are highly correlated, the relevance of each of them was tested in separate models.

\subsubsection{Procedure}

The procedure was the same as in Experiments 2 and 4. Note again that all stimuli were presented at $80 \mathrm{~dB}$ SPL for all listeners.

\subsection{Results and discussion}

As in Experiments 2, 3 and 4, we only analysed responses that we knew were either correct or incorrect (this led to the exclusion of $5 \%$ of the responses). In Fig. 7, accuracy rates of the older listeners are given for the target words in the two context conditions, broken down by degree of reduction. Fig. 8 shows the combined results of the auditory isolation and auditory context conditions for the three listener groups of Experiments 2, 4 and 5 .

We first compared the results of the older listeners to those of the young normal-hearing listeners (Experiment 2). As before, the

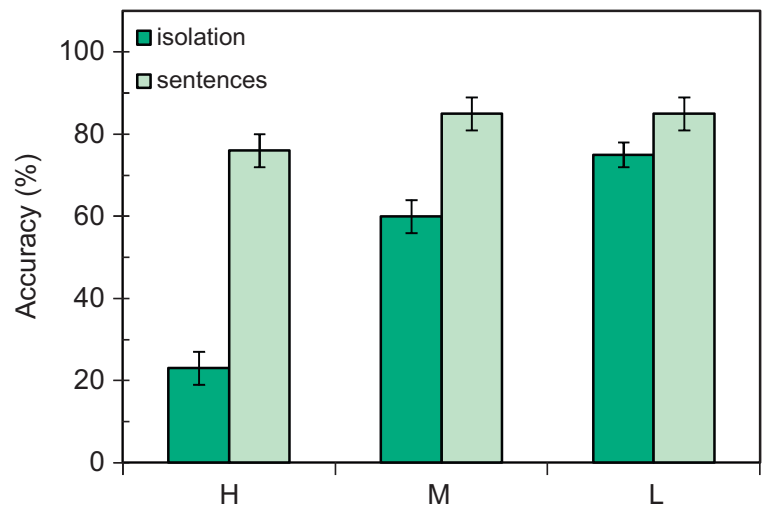

Fig. 7. Results of the older participants: accuracy rates for the words of varying degrees of reduction (High, Medium and Low) in the two context conditions (in isolation or in sentences).

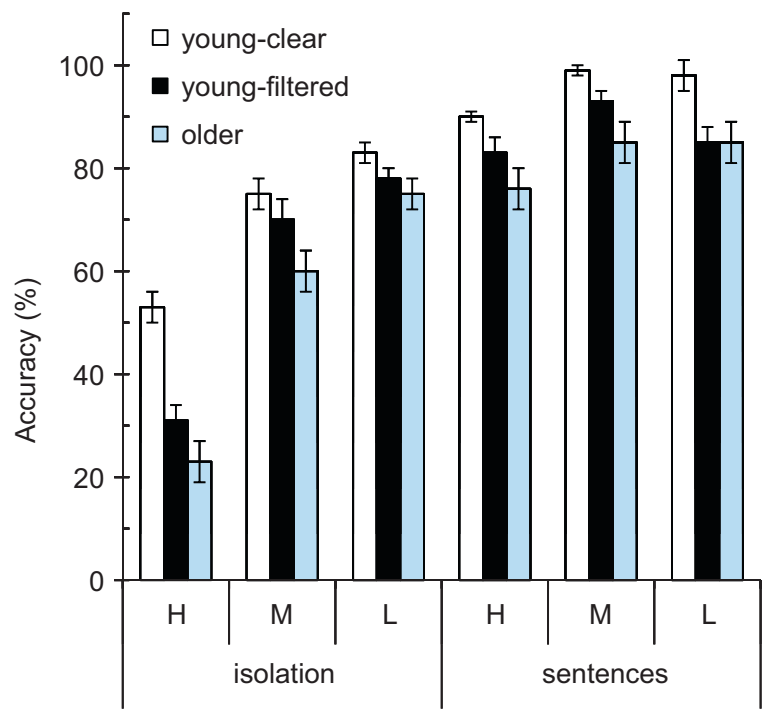

Fig. 8. Results of all three listener groups of Experiments 2, 4 and 5 (young adults, young adults who were presented with filtered speech, and the older adults): accuracy rates for the words of varying degrees of reduction (High, Medium and Low) in the two context conditions (in isolation or in their auditory contexts).

responses (2358 observations altogether) were analysed with a multi-level regression analysis for binomial data (the dependent variable being whether a response was correct or incorrect) with participant and word as crossed grouping factors. Factors were Age Group, Degree of Reduction of the word and the Context in which it was presented. All three factors showed significant effects. The significant effects and interactions are given in Table 1.

Older participants showed poorer identification performance than the young normal-hearing listeners. Furthermore, as in the analysis of Experiment 2, words were better recognised in their (auditory) sentential contexts than in isolation; and, relative to items of High reduction, the items of Medium reduction and those of Low reduction were better identified. We again found a significant interaction between Context and Degree of Reduction. Importantly, whereas the Context by Age Group interaction was not significant, the three-way interaction between Context, Degree of Reduction and Age Group was. While the two listener groups did not differ in the context effect for highly reduced words, the context benefit on recognition was smaller for the older adults than for the young adults for words with Medium reduction and words with Low reduction. We confirmed our 
Table 1

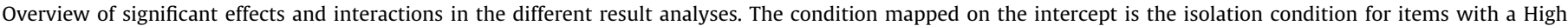
degree of reduction (young adults).

\begin{tabular}{|c|c|c|c|c|}
\hline Effect & $\beta$ & $S E$ & $z$ & $\boldsymbol{p}$ \\
\hline \multicolumn{5}{|l|}{ Older adults vs. young listeners (Experiment 5 vs. Experiment 2) } \\
\hline Context & 2.08 & 0.46 & 4.50 & $<0.001$ \\
\hline Degree of Reduction (Medium) & 0.86 & 0.28 & 3.13 & $<0.01$ \\
\hline Degree of Reduction (Low) & 1.61 & 0.29 & 5.56 & $<0.001$ \\
\hline Participant group (older adults) & -1.82 & 0.32 & -5.69 & $<0.001$ \\
\hline Context $\times$ Degree of Reduction $(\mathrm{M})$ & -2.63 & 1.11 & -2.36 & $<0.05$ \\
\hline Participant group $\times$ Context $\times$ Degree of Reduction (Medium) & -3.23 & 1.17 & 2.77 & $<0.01$ \\
\hline Participant group $\times$ Context $\times$ Degree of Reduction (Low) & -2.56 & 0.82 & -3.13 & $<0.01$ \\
\hline \multicolumn{5}{|c|}{ Older adults vs. young listeners who heard filtered speech (Experiment 5 vs. Experiment 4) } \\
\hline Context & 2.85 & 0.37 & 7.78 & $<0.001$ \\
\hline Degree of Reduction (Medium) & 1.86 & 0.21 & 8.91 & $<0.001$ \\
\hline Degree of Reduction (Low) & 2.84 & 0.21 & 13.59 & $<0.001$ \\
\hline Context $\times$ Degree of Reduction (Low) & -2.08 & 0.31 & -6.78 & $<0.001$ \\
\hline \multicolumn{5}{|l|}{ Individual differences among the older adults } \\
\hline Context & 3.57 & 0.53 & 6.79 & $<0.001$ \\
\hline Degree of Reduction (Medium) & 1.86 & 0.28 & 6.62 & $<0.001$ \\
\hline Degree of Reduction (Low) & 2.95 & 0.28 & 10.42 & $<0.001$ \\
\hline Context $\times$ Degree of Reduction (Low) & -1.82 & 0.42 & -4.31 & $<0.001$ \\
\hline Hearing loss & -0.04 & 0.01 & -4.49 & $<0.001$ \\
\hline Residual-effect-of-age & -0.07 & 0.02 & -2.95 & $<0.01$ \\
\hline Context $\times$ Hearing loss & -0.02 & 0.01 & -2.26 & $<0.05$ \\
\hline
\end{tabular}

interpretation of this three-way interaction by separately testing item subsets (breaking items down by the three Degrees of Reduction) for the Age Group by Context interaction. For the items of Low and Medium reduction, Age Group and Context showed a significant interaction: for both item subsets, the older adults benefited less from context than the young adults (Low reduction items: $\beta=-1.83$ ( $S E=0.82$ ), $z=-2.23, p<0.05$; and Medium reduction items: $\beta=-2.48(S E=1.13), z=-2.20, p<0.05)$. For the items in the High reduction group, older adults and young benefited equally from context. These subset analyses replicated the pattern we found in the comparison of normal-hearing young adults with the young adults with simulated hearing loss (Experiments 2 and 4). As before, the statistical model gave a significantly better fit if Context was added to the random item part (loglikelihood ratio test $\left.\chi^{2}(2)=62.154, p<0.001\right)$.

We then compared the results of the older listeners to those of the young adults who had been presented with the filtered speech (Experiment 4). Apart from Context and Degree of Reduction, we also tested whether Age Group had an effect. The significant effects and interactions in this comparison are also given in Table 1. This model replicated the effects of Context and Degree of Reduction, and their interaction. As before, the statistical model gave a significantly better fit if Context was added to the random item part ( $\log$ likelihood ratio test $\left.\chi^{2}(2)=64.489, p<0.001\right)$. There was no overall effect of Age Group, and Age Group did not interact with either of the two factors. This means that simulation of an age-related hearing loss brought young participants to about the same performance level as the older participants. Furthermore, the pattern of context use across the different degrees of reduction is similar for the two hearing-impaired age groups, as can be seen from Fig. 8.

Finally, we also analysed the results of the older adults separately to investigate whether individual characteristics, such as hearing loss, would predict performance and context use among the older adults. Apart from the factors manipulated in the study (Degree of Reduction and Context), several other predictors were entered into the statistical model of the older listeners' data: the participant's gender, hearing loss, age, and the measures derived from the Stroop task (average neutral naming speed, average naming speed in the incongruent condition, and an interference value, being the ratio of these two). Because age and hearing loss are correlated in this participant group (Pearson correlation coefficient for this group was $R=0.463, p=0.010$ ), we orthogonalised the two predictors by replacing Age by the residuals of a linear regression model predicting Age as a function of hearing loss.

As can be seen from Table 1, this model replicated the earlier effects for Context, Degree of Reduction, and their interaction. In addition, hearing loss and the residual-effect-of-Age affected performance: the more hearing loss, the poorer overall identification performance, and also the older the participant, the poorer performance. Importantly, Context interacted with hearing loss: the more hearing loss the participant had, the smaller the beneficial effect of context.

A participant's average colour-naming speed in the Stroop neutral (XXXX) condition, which can be taken as a measure of information processing speed, did not predict performance in the identification task, nor did average naming time in the incongruent condition, nor the interference combination measure. Thus, the overall Age effect on performance (cf. also Jerger et al., 1991; van Rooij \& Plomp, 1990, 1992; van Rooij et al., 1989) may result from some type of age-related cognitive decline that is not captured by the Stroop task. Measures of cognitive function other than a measure of inhibitory abilities might have been a better choice. Since wider context is needed to resolve ambiguous words, age-related declines in verbal working memory could turn out to be important (Wingfield, Alexander, \& Cavigelli, 1994). These issues remain for further investigation.

In conclusion, the pattern of results observed for the older adults was very similar to that obtained with the young adults with a simulated hearing loss. The two 'hearing-impaired' groups showed equally poor recognition of reduced words presented out of context. Moreover, both groups benefited less from information contained in a word's context than normal-hearing adults, except for the most reduced forms. This effect of poorer hearing on context use is supported by our finding that among the older participants, those with more hearing loss benefited less from context.

Interestingly, the difference in context benefit between the hearing-impaired and normal-hearing listeners only manifests itself for the words with Low and Medium reduction. We will come back to this issue in the Section "Discussion". 


\section{Discussion}

The aim of this study was twofold. First, we investigated the role of acoustic properties of reduced words for word recognition. Second, we investigated the role of information contained in a word's context for spoken-word recognition, being semantic/ syntactic (top-down) information, as well as acoustic cues to the target word.

The results of Experiments 2 through 5 speak to the first issue. Reduced word forms presented out of context were repeatedly shown to be more difficult to process than less reduced forms: the degree of reduction of the target word affected target intelligibility in all experiments. Further, the comparisons between listener groups of Experiments 2, 4 and 5 emphasised the importance of good hearing in bottom-up processing of the speech forms.

Importantly, the results of Experiment 3 showed that participants can be trained to recognise reduced target words excised out of their auditory context: target recognition accuracy in the isolation condition was higher in Experiment 3, where participants listened to isolated reduced words throughout the experiment, than in Experiment 2. The young listeners of Experiment 3 seem to get more (acoustic) information out of these short fragments in the second half of the experiment because they have had more training (which includes information about which words are likely to occur in the experiment). This is in line with our finding that the older adults (Experiment 5) showed similar performance to the young adults who heard the filtered speech (Experiment 4), despite the fact that the hearing loss simulation in the young adults was an underestimation of the older adults' hearing problems in terms of simulated sensitivity (cf. Fig. 6), and because the filtering did not simulate the distortion caused by sensorineural hearing loss. These results suggest that older adults might have outperformed the young adults if the two groups had been accurately matched on hearing abilities. As suggested by Stelmachowicz et al. (1990), older adults may perform relatively better because they have learned to pay more attention to lowfrequency information.

The second issue, the role of context information for word recognition in conversational speech, is addressed in several ways in this study. Experiment 1, a visual cloze test, showed that most target words from the Ernestus et al. (2002) study could not be guessed purely from their contexts. Experiment 2 replicated the Ernestus et al. (2002) finding that presentation of the target words in their auditory contexts strongly increased target recognition accuracy (from 53\% to $90 \%$ for highly reduced words). Importantly, Experiment 3, which combined the auditory presentation of the excised target words with the visual (orthographic) presentation of their sentence contexts, showed that the contexts did contain some semantic/syntactic constraints, as the presentation of visual context also improved auditory target word recognition. These results demonstrate that the semantic/syntactic cues are by themselves of little help, but they can constrain the number of words that fit the acoustic signal. The weak constraints interact with the acoustic properties of the reduced target words to facilitate their recognition. The results of Experiment 3 also provided evidence that the auditory context results in our Experiments 2, 4 and 5 are to some extent due to acoustic cues in the context, because presenting the target words in their auditory context boosted recognition more than presenting them with their context in a visual form. In the Introduction we suggested the possibility that contextual (acoustic) information in a less formal speech style could be more difficult to pick up than in read speech because conversational speech can be articulated very quickly. The fact that participants got more information out of the auditory context in Experiment 2 than out of the visual context in Experiment 3 to facilitate target word recognition is all the more interesting considering that Experiment provided a very clear orthographic representation of the target word's semantic/syntactic context (whereas information concerning neighbouring words and thus semantic/syntactic context was transient and less clear in Experiment 2). The present results therefore clearly demonstrate that contextual acoustic cues play an important role for spoken-word recognition in conversational speech.

Further, Experiments 4 and 5 showed that a substantial part of the contextual information is carried by the high-frequency part of the speech signal. Attenuation of high frequencies led to a reduced context benefit on word recognition. The reduced context benefit was evidenced by three findings: (1) compared to the normal-hearing young adults, the young adults in whom we simulated a hearing impairment benefited less from context; (2) the older participants benefited less from context than the young normal-hearing adults; and (3) among the older listeners, we found that the more hearing-impaired participants were, the less benefit they gained from context. These context findings complement the robust finding of older listeners' increased reliance on semantic context (Nittrouer \& Boothroyd, 1990; Pichora-Fuller et al., 1995; Sheldon, Pichora-Fuller, \& Schneider, 2008; Sommers \& Danielson, 1999; Wingfield, Aberdeen, \& Stine, 1991). In most of these semantic context studies, standardised Sentence-Perception-In-Noise materials (SPIN-R) were used in which the sentence-final word is either highly predictable from the preceding semantic context, or has a low predictability (Bilger, Neutzel, \& Rzeczowski, 1984; Kalikow, Stevens, \& Elliott, 1977). When equated for identification performance in the lowprobability condition (by having different signal-to-noise ratios), subjects with age-related hearing loss benefited more from context in the high-probability condition than old subjects with near-normal hearing, who in turn benefited more from context than young (normal-hearing) subjects (Pichora-Fuller et al., 1995). Pichora-Fuller et al. (1995) found this 'greater context benefit' pattern over a range of signal-to-noise ratios and thus over a range of performance levels. In our study, recognition performance in the isolation condition was not equated across groups. This allowed us to investigate which sources of information are available if the input is exactly the same for all participants. The information contained in our auditory context fragments, semantic/syntactic and acoustic cues, may not be as helpful to hearing-impaired listeners as strongly biasing semantic context. A reduced benefit from acoustic context may in fact add to older listeners' increased reliance on semantic context and topdown processing.

Interestingly, contextual benefit for normal-hearing and hearing-impaired listeners was equal for the Highly reduced words. This first of all demonstrates that a smaller context benefit for adults with hearing impairment for the words with Low and Medium reduction should not be attributed to a floor effect (i.e., overall lower performance on our test materials). There are at least two possible explanations for the finding of equal benefit for the Highly reduced words. First, Highly reduced words are articulatorily weak which may imply a higher degree of coarticulation with neighbouring words. Thus, Highly reduced words may have more acoustic cues in their context than Low/Medium reduced words. The more acoustic cues to the target, the more likely it is that they are picked up by hearing-impaired listeners. Second, hearing-impaired listeners may (unconsciously) refrain from using acoustic contextual information, unless word identification without using acoustic context is almost impossible. Acoustic cues in neighbouring words may be subtle and difficult to interpret, given the high variability in conversational speech, 
particularly for hearing-impaired listeners. As a consequence, hearing-impaired listeners mainly use acoustic context information for recognition of the difficult Highly reduced words, which they identify correctly without context in only $23 \%$ (older adults) or $31 \%$ (young adults with simulated hearing impairment) of the cases. Further research is required to investigate these two possibilities.

The results of our study, particularly the different recognition scores of words in auditory (Experiment 2) and visual (Experiment 3 ) contexts, suggest that acoustic cues in a word's context are more useful than previously assumed in models of spokenword recognition (e.g., Gaskell \& Marslen-Wilson, 1998; McClelland \& Elman, 1986; Norris, 1994; Norris et al., 2000), and fits in with the idea that the spreading of acoustic cues over a target word's neighbouring syllables makes the speech signal perceptually coherent (Hawkins, 2003) and that this perceptual coherence is the 'perceptual glue' of speech (Remez, 2003). It is also in line with the 'perceptual grouping hypothesis' proposed in Dilley and McAuley (2008) that "prosodic (i.e., fundamental frequency and duration) cues distal from the locus of segmentation or lexical access of a word affect the unfolding process of perceiving prosodic constituents, thereby influencing word segmentation and lexical recognition in a manner consistent with principles of perceptual organisation for non-speech auditory patterns" (p. 296).

A first step towards modelling the role of near phonetic context for spoken-word recognition was made in papers on place assimilation and deletion of segments (e.g., Gaskell, 2003; Gaskell \& Snoeren, 2008; Gaskell \& Marslen-Wilson, 1996, 1998; Mitterer, Yoneyama, \& Ernestus, 2008). Hawkins and Smith (2001), in their POLYSP model (for 'polysystemic speech understanding'), focus on the role of systematic fine phonetic detail that may be spread throughout the speech signal and how it facilitates analysis at all linguistic levels simultaneously. This integration of information over longer time scales may not be easily captured in recurrent neural networks (Hawkins \& Smith, 2001). However, future models of speech perception cannot afford to ignore these wider-range bottom-up acoustic effects.

In conclusion, the current results stress the importance of acoustic information in a reduced word and in its context for recognition, and have provided some initial data on the importance of the high-frequency spectrum. Our results also suggest that listeners can be trained to get more out of a spectrally impoverished speech signal by paying more attention to information in other parts of the spectrum. Finally, we have shown that semantic/syntactic information contributes to word recognition, particularly in combination with acoustic information in the reduced word.

\section{References}

Baayen, R. H., Davidson, D. J., \& Bates, D. M. (2008). Mixed-effects modelling with crossed random effects for subjects and items. Journal of Memory and Language, 59, 390-412.

Bilger, R. C., Neutzel, J. M., \& Rzeczowski, C. (1984). Standardisation of a test of speech perception in noise. Journal of Speech and Hearing Research, 27, 32-48.

Coleman, J. (2003). Discovering the acoustic correlates of phonological contrasts. Journal of Phonetics, 31, 351-372.

Craig, C. H. (1992). Effects of aging on time-gated isolated word-recognition performance. Journal of Speech and Hearing Research, 35, 234-238.

Craig, C. H., Kim, B. W., Rhyner, P. M. P. \& Chirillo, T. K. B. (1993). Effects of word predictability, child development, and aging on time-gated speech recognition performance. Journal of Speech and Hearing Research, 36, 832-841.

Dilley, L. C., \& McAuley, J. D. (2008). Distal prosodic context affects word segmentation and lexical processing. Journal of Memory and Language, 59, 294-311.

Ellis, L., \& Hardcastle, W. J. (2002). Categorical and gradient properties of assimilation in alveolar to velar sequences: Evidence from EPG and EMA data. Journal of Phonetics, 30, 373-396.
Ernestus, M. (2000). Voice assimilation and segment reduction in casual Dutch, a corpus-based study of the phonology-phonetics interface. Utrecht: LOT.

Ernestus, M., \& Baayen, R. H. (2007). The comprehension of acoustically reduced morphologically complex words: The roles of deletion, duration and frequency of occurrence. In Proceedings of the 16th international congress of phonetic sciences (pp. 773-776). Saarbruecken, Germany.

Ernestus, M., Baayen, H., \& Schreuder, R. (2002). The recognition of reduced word forms. Brain and Language, $81,162-173$.

Garner, W. R. (1974). The processing of information and structure. Potomac, MD (USA): Erlbaum

Gaskell, M. G. (2003). Modeling regressive and progressive effects of assimilation in speech perception. Journal of Phonetics, 31, 447-463.

Gaskell, M. G., \& Marslen-Wilson, W. D. (1996). Phonological variation and inference in lexical access. Journal of Experimental Psychology: Human Perception and Performance, 22, 144-158.

Gaskell, M. G., \& Marslen-Wilson, W. D. (1998). Mechanisms of phonological inference in speech perception. Journal of Experimental Psychology: Human Perception and Performance, 24, 380-396.

Gaskell, M. G., \& Snoeren, N. (2008). The impact of strong assimilation on the perception of connected speech. Journal of Experimental Psychology: Human Perception and Performance, 34, 1632-1647.

Gow, D. W. (2001). Assimilation and anticipation in continuous spoken word recognition. Journal of Memory and Language, 45, 133-159.

Gow, D. W. (2002). Does English coronal place assimilation create lexical ambiguity?. Journal of Experimental Psychology: Human Perception and Performance, 28, 163-179.

Hargus, S. E., \& Gordon-Salant, S. (1995). Accuracy of speech intelligibility index predictions for noise-masked young listeners with normal hearing and for elderly listeners with hearing impairment. Journal of Speech and Hearing Research, 38, 234-243.

Hawkins, S. (2003). Roles and representations of systematic fine phonetic detail in speech understanding. Journal of Phonetics, 31, 373-405.

Hawkins, S., \& Smith, R. (2001). Polysp: A polysystemic, phonetically rich approach to speech understanding. Italian Journal of Linguistics - Rivista di Linguistica, 13, 99-188.

Heid, S., \& Hawkins, S. (2000). An acoustical study of long-domain /r/ and /1/ coarticulation. In Proceedings of the fifth seminar on speech production: Models and data (ISCA) (pp. 77-80). Kloster Seeon, Bavaria, Germany.

Humes, L. E., Dirks, D. D., Bell, T. S., \& Kincaid, G. E. (1987). Recognition of nonsense syllables by hearing-impaired listeners and by noise-masked normal hearers. Journal of the Acoustical Society of America, 81, 765-773.

Humes, L. E., Lee, J. H., \& Coughlin, M. P. (2006). Auditory measures of selective and divided attention in young and older adults using single-talker competition. Journal of the Acoustical Society of America, 120, 2926-2937.

Janse, E. (2004). Word perception in fast speech: Artificially time-compressed vs. naturally produced fast speech. Speech Communication, 42, 155-173.

Janse, E. (2009). Processing of fast speech by elderly listeners. Journal of the Acoustical Society of America, 125, 2361-2373.

Janse, E., Nooteboom, S., \& Quené, H. (2007). Coping with gradient forms of /t/deletion and lexical ambiguity in spoken word recognition. Language and Cognitive Processes, 22, 161-200.

Jerger, J., Jerger, S., \& Pirozzolo, F. (1991). Correlational analysis of speech audiometric scores, hearing loss, age, and cognitive abilities in the elderly. Ear and Hearing, 12, 103-109.

Johnson, K. (2004). Massive reduction in conversational American English. In K. Yoneyama, \& K. Maekawa (Eds.), Spontaneous speech: Data and analysis. Proceedings of the 1st session of the 10th international symposium (pp. 29-54). Tokyo, Japan: The National International Institute for Japanese Language.

Kalikow, D. N., Stevens, K. N., \& Elliott, L. L. (1977). Development of a test of speech intelligibility in noise using sentence materials with controlled word predictability. Journal of the Acoustical Society of America, 6l, 1337-1361.

Kelly, J., \& Local, J. K. (1986). Long domain resonance patterns in English. In International conference on speech input/output: Techniques and applications (pp. 304-309). Conference Publication No. 258. London: Institute of Electrical Engineers.

Kemps, R., Ernestus, M., Schreuder, R., \& Baayen, R. H. (2004). Processing reduced word forms: The suffix restoration effect. Brain and Language, 19, 117-127.

Local, J. (2003). Variable domains and variable relevance: Interpreting phonetic exponents. Journal of Phonetics, 31, 321-339.

McClelland, J. L., \& Elman, J. L. (1986). The TRACE model of speech perception. Cognitive Psychology, 18, 1-86.

Mitterer, H., Yoneyama, K., \& Ernestus, M. (2008). How we hear what is hardly there: Mechanisms underlying compensation for $/ \mathrm{t} /$-reduction in speech comprehension. Journal of Memory and Language, 59, 133-152.

Nittrouer, S., \& Boothroyd, A. (1990). Context effects in phoneme and word recognition by young children and older adults. Journal of the Acoustical Society of America, 87, 2705-2715.

Nolan, F. (1992). The descriptive role of segments: Evidence from assimilation. In G. J. Docherty, \& D. R. Ladd (Eds.), Papers in laboratory phonology II (pp. 261280). Cambridge: Cambridge University Press.

Norris, D. (1994). Shortlist: A connectionist model of continuous speech recognition. Cognition, 52, 189-234

Norris, D., McQueen, J. M., \& Cutler, A. (2000). Merging information in speech recognition: Feedback is never necessary. Behavioral and Brain Sciences, 23, 299-370. 
Payton, K. L., Uchanski, R. M., \& Braida, L. D. (1994). Intelligibility of conversational and clear speech in noise and reverberation for listeners with normal and impaired hearing. Journal of the Acoustical Society of America, 95, 1581-1592.

Picheny, M. A., Durlach, N. I., \& Braida, L. D. (1985). Speaking clearly for the hard of hearing. I. Intelligibility differences between clear and conversational speech. Journal of Speech and Hearing Research, 28, 96-103.

Picheny, M. A., Durlach, N. I., \& Braida, L. D. (1986). Speaking clearly for the hard of hearing. II. Acoustic characteristics of clear and conversational speech. Journal of Speech and Hearing Research, 29, 434-446.

Picheny, M. A., Durlach, N. I., \& Braida, L. D. (1989). Speaking clearly for the hard of hearing. III. An attempt to determine the contribution of speaking rate to differences in intelligibility between clear and conversational speech. Journal of Speech and Hearing Research, 32, 600-603.

Pichora-Fuller, M. K., Schneider, B., \& Daneman, M. (1995). How young and old listeners listen to and remember speech in noise. Journal of the Acoustical Society of America, 97, 593-608.

Pinheiro, J. C., \& Bates, D. M. (2000). Mixed-effects models in S and S-plus. New York: Springer.

Pickett, J. M., \& Pollack, I. (1963). Intelligibility of excerpts from fluent speech: Effects of rate of utterance and duration of excerpt. Language E Speech, 3, 151-164.

Quené, H., \& van den Bergh, H. (2004). On multi-level modeling of data from repeated measures designs: A tutorial. Speech Communication, 43, 103-121.

Ranbom, L. J., \& Connine, C. M. (2007). Lexical representation of phonological variation in spoken word recognition. Journal of Memory and Language, 57, 273-298.

Remez, R. E. (2003). Establishing and maintaining perceptual coherence: Unimodal and multimodal evidence. Journal of Phonetics, 31, 293-304.

van Rooij, J. C. G. M., \& Plomp, R. (1990). Auditive and cognitive factors in speech perception by elderly listeners. II. Multivariate analyses. Journal of the Acoustical Society of America, 88, 2611-2624.

van Rooij, J. C. G. M., \& Plomp, R. (1992). Auditive and cognitive factors in speech perception by elderly listeners. III. Additional data and final discussion. Journal of the Acoustical Society of America, 91, 1028-1033.

van Rooij, J. C. G. M., Plomp, R., \& Orlebeke, J. F. (1989). Auditive and cognitive factors in speech perception by elderly listeners. I. Development of test battery. Journal of the Acoustical Society of America, 86, 1294-1309.

Salthouse, T. A. (1996). The processing speed theory of adult age differences in cognition. Psychological Review, 103, 403-428.

Salverda, A. P., Dahan, D., \& McQueen, J. M. (2003). The role of prosodic boundaries in the resolution of lexical embedding in speech comprehension. Cognition, 90 51-89.
Shatzman, K. B., \& McQueen, J. M. (2006). Segment duration as a cue to word boundaries in spoken-word recognition. Perception \& Psychophysics, 68, 1-16.

Sheldon, S., Pichora-Fuller, M. K., \& Schneider, B. A. (2008). Priming and sentence context support listening to noise-vocoded speech by younger and older adults. Journal of the Acoustical Society of America, 123, 489-499.

Sommers, M. S., \& Danielson, S. E. (1999). Inhibitory processes and spoken word recognition in young and older adults: The interaction of lexical competition and semantic context. Psychology and Aging, 14, 458-472.

Sommers, M. S., Nygaard, L. C., \& Pisoni, D. B. (1994). Stimulus variability and spoken word recognition. I. Effects of variability in speaking rate and overall amplitude. Journal of the Acoustical Society of America, 96, 1314-1324.

Spinelli, E., McQueen, J. M., \& Cutler, A. (2003). Processing resyllabified words in French. Journal of Memory and Language, 48, 233-254.

Stelmachowicz, P. G., Lewis, D. E., Kelly, W. J., \& Jesteadt, W. (1990). Speech perception in low-pass filtered noise for normal and hearing-impaired listeners. Journal of Speech and Hearing Research, 33, 290-297.

Stroop, J. R. (1935). Studies of interference in serial verbal reactions. Journal of Experimental Psychology, 18, 643-661.

Swinney, D. (1979). Lexical access during sentence comprehension: (Re)consideration of context effects. Journal of Verbal Learning and Verbal Behavior, 18 645-659.

Tucker, B. V., \& Warner, N. (2007). Inhibition of processing due to reduction of the American English flap. In Proceedings of the 16th international congress of phonetic sciences (pp. 1949-1952). Saarbruecken, August 2007.

Tun, P. A., O'Kane, G., \& Wingfield, A. (2002). Distraction by competing speech in young and older adult listeners. Psychology and Aging, 17, 453-467.

Tunley, A. (1999). Coarticulatory influences of liquids on vowels in English. Unpublished Ph.D. Dissertation. University of Cambridge.

Uchanski, R. M., Choi, S., Braida, L. D., Reed, C. M., \& Durlach, N. I. (1996). Speaking clearly for the hard of hearing. IV. Further studies of the role of speaking rate. Journal of Speech and Hearing Research, 39, 494-509.

West, P. (2000). Perception of distributed coarticulatory properties of English /1/ and /Eे/. Journal of Phonetics, 27, 405-425.

Wingfield, A., Aberdeen, J. S., \& Stine, E. A. (1991). Word onset gating and linguistic context in spoken word recognition by young and elderly adults. Journal of Gerontology, 46, 127-129.

Wingfield, A., Alexander, A. H., \& Cavigelli, S. (1994). Does memory constrain utilization of top-down information in spoken word recognition? Evidence from normal aging. Language and Speech, 37, 221-235. 